\title{
Indexed maximal left atrial volume predicts response to cardiac resynchronization therapy
}

\author{
Luca Rossi a, $^{\text {, }}$, Alessandro Malagoli a,*, Massimo Piepoli ${ }^{\text {a,2 }}{ }^{\text {, Francesco Franchi b,3, Vincenzo Malavasi }}{ }^{\text {b, }}$, \\ Edoardo Casali ${ }^{\text {b,5 }}$, Guido Rusticali ${ }^{\text {a,6 }}$, Giovanni Quinto Villani ${ }^{\text {a,6 }}$ \\ a Department of Cardiology, “Guglielmo da Saliceto” Hospital, Piacenza, Via Taverna 49, 29100 Piacenza, Italy \\ b Department of Cardiology, Policlinico Hospital, Modena and Reggio Emilia University, Modena, Italy
}

\section{A R T I C L E I N F O}

\section{Article history:}

Received 19 September 2012

Received in revised form 29 January 2013

Accepted 4 May 2013

Available online 29 May 2013

\section{Keywords:}

Left atrial volume

Cardiac resynchronization therapy (CRT)

Chronic heart failure

\begin{abstract}
A B S T R A C T
Aims: Cardiac resynchronization therapy (CRT) has shown morbidity and mortality benefits in patients with advanced congestive heart failure (HF). Since about one-third of the patients did not appear to respond to CRT, it would seem reasonable to try to identify patients more accurately before implantation. Left atrial (LA) dimension has been proposed as a powerful outcome predictor in patients with heart disease. Accordingly, the aim of this study is to prospectively assess the predictive value of LA for selecting CRT responders.

Methods: Fifty two consecutive patients with refractory HF, sinus rhythm and left bundle branch block were enrolled in the study and planned for CRT implantation. Clinical and echocardiographic evaluations were performed before CRT implantation and after 6 months. Three LA volumes indexed to body surface area (iLAV) were computed to evaluate the LA complexity: maximal LAV (iLAVmax), LAV just before atrial systole (iLAVpre), and minimal LAV (iLAVpost). CRT responders were defined as those who presented a reduction of $>10 \%$ in LVESVi at 6-month follow-up.

Results: Responders (63\%) and nonresponders (37\%) had similar baseline clinical characteristics and preimplantation LV volumes. However, baseline LA volumes were significantly associated with the extent of LV reverse remodeling: in particular, baseline iLAVmax was remarkably lower in responders than in nonresponders $\left(50.2 \pm 14.1 \mathrm{ml} / \mathrm{m}^{2}\right.$ vs $\left.65.8 \pm 15.7 \mathrm{ml} / \mathrm{m}^{2}, p=0.001\right)$ resulting predictive for CRT response.

Conclusion: Patients with small iLAV result as better responders to CRT than larger one. iLAVmax is an independent predictor of LV reverse remodeling and allows to indentify the best candidates for CRT.
\end{abstract}

(c) 2013 Elsevier Ireland Ltd. All rights reserved.

\section{Introduction}

Cardiac resynchronization therapy (CRT) has become an effective treatment modality in patients with advanced congestive heart failure (HF) and prolongs survival compared to optimal medical therapy alone $[1,2]$. However, about $30 \%$ of patients fail to respond to this therapy, realizing a growing interest toward the identification of potential responders to CRT before implantation [3]. Recently, several echocardiographic measurements of ventricular dyssynchrony have been tested for prediction of CRT response with mixed results.

\footnotetext{
* Corresponding author. Tel.: + 390523 303221; fax: + 390523303220

E-mail addresses: rossi_luca@alice.it (L. Rossi), ale.malagoli@gmail.com (A. Malagoli),m.piepoli@ausl.pc.it (M. Piepoli), francesco.franchi@libero.it (F. Franchi), nanni.malavasi@gmail.com (V. Malavasi), edoardocasali@libero.it (E. Casali), g.rusticali@ausl.pc.it (G. Rusticali), g.villani@ausl.pc.it (G.Q. Villani).

1 Tel.: + 390523 303221; fax: + 390523303220 .

2 Tel.: + 390523 303222; fax: + 390523303220 .

3 Tel.: + 39059 4225459; fax: + 390594224323 .

4 Tel: + 39059 4225463; fax: + 390594224323 .

5 Tel.: + 39059 4225462; fax: + 390594224323.

6 Tel.: + 390523 303215; fax: + 390523303220
}

To date the role of left atrium (LA) to predict response to CRT has not yet been evaluated, although LA size has been suggested as a powerful outcome predictor in patients with heart disease [4-6]. Moreover, LA size expresses the chronicity of exposure to abnormal filling pressures and has been proposed in a retrospective analysis as a predictor of mortality after CRT in patients with advanced HF [7]. Accordingly, the purpose of the present study was to prospectively assess the predictive value of LA for selecting CRT responders.

\section{Methods \\ 2.1. Patient selection and study protocol}

From the Cardiology Department of Piacenza Hospital and Modena University Hospital fifty two consecutive patients with refractory HF, left bundle branch block and sinus rhythm were prospectively enrolled in the study before CRT implantation. Patients were classified as having HF of ischemic or nonischemic etiology based on a history of myocardial infarction or based on objective evidence of coronary artery disease as assessed with coronary angiography. The criteria for CRT implantation were based according to the established international guidelines: symptomatic patients (NYHA functional class II or more), left ventricular ejection fraction (LVEF) less than 35\%, QRS duration $>120 \mathrm{~ms}$ ( $>150 \mathrm{~ms}$ if NYHA functional class II) although on optimal pharmacologic therapy, including diuretics, $\beta$-blockers, and angiotensin converting enzyme inhibitors or angiotensin receptor 
blockers for at least 30 days prior to enrollment in the study. Exclusion criteria were symptomatic bradyarrhythmias, previous history of atrial arrhythmias, pregnancy, myocardial infarction or coronary intervention within 3 months of enrollment, or a significant comorbid illness defined as severe obstructive pulmonary disease requiring chronic supplementation of oxygen, severe renal failure (creatinine clearance $<30 \mathrm{ml} / \mathrm{min}$ ), malignancy, or medically refractory anginal symptoms.

Patients meeting inclusion criteria underwent a baseline evaluation prior to CRT that included NYHA functional classification, quality-of-life (QOL) assessment with the Minnesota Living with Heart Failure Questionnaire and resting two-dimensional Doppler echocardiographic study. At 6-month follow-up the same echocardiographic and clinical parameters were reassessed. The study complies with the Declaration of Helsinki, the study protocol was approved by the regional ethics committee of our institutions, and informed consent was obtained from each patient.

\subsection{Device implantation}

All fifty two patients underwent successful implantation of a biventricular device. The LV pacing lead was inserted by a transvenous approach through the coronary sinus to target lateral or posterolateral cardiac vein; in one patient the target vein was unavailable, so anterior vein was used. One day after implantation, the LV lead position was assessed from a chest X-ray using the lateral views. The right atrial and ventricular leads were positioned conventionally in right atrial appendage and in right ventricular apex respectively. Choices of CRT devices included biventricular defibrillators in 42 patients (Concerto II from Medtronic Inc.; Contak Renewal 4 from Boston scientific; Atlas II HF from St. Jude Medicals) and biventricular pacemakers in 10 patients (InSync III from Medtronic Inc.; Contak Renewal TR2 from Boston scientific; Frontier II from St. Jude Medicals). No AVD dynamic mode was set and no AV delay optimization guided by echocardiography was performed after device implantation.

\subsection{Echocardiography}

Transthoracic echocardiography was performed with the subjects at rest in the left lateral position with commercially available ultrasound equipment (Vivid 7, General Electric and Acuson Sequoia c512, Siemens Medical). Images were obtained using a 3.5-MHz transducer, at a depth of $16 \mathrm{~cm}$ in the parasternal and apical views (standard long-axis, two-chamber and four-chamber images) and measurements were performed after a 10-minute run-in period for stabilization and equilibration.

\subsection{1. $L V$ and mitral valve evaluation}

The LV volumes and the LVEF were calculated from the apical two- and four-chamber images using the biplane Simpson's rule. Values were indexed to body surface area calculated using the Du Bois and Du Bois Formula [8]. LV reverse remodeling was computed as LV end-systolic volume (LVESVi) change between baseline and 6-month follow-up values ( $\triangle$ LVESVi). Inter-ventricular mechanical delay (IVMD) was calculated as the absolute difference in the LV and right ventricular pre-ejection times, with $\geq 40 \mathrm{~ms}$ IVMD regarded as a significant delay. LV dyssynchrony was assessed by septal-to-lateral delay (Ts-LS) defined as described previously [9]. The ratio between peak early (E) and late (A) diastolic LV filling velocities was used as standard indices of LV diastolic function [10]. LV longitudinal function was explored by pulsed Tissue Doppler imaging, placing the sample volume at the level of mitral septal annulus from the apical fourchamber view [11]. Mean peak early diastolic ( $\left.E^{\prime}\right)$ annular velocities were measured for 3 consecutive beats and averaged. Mean $\mathrm{E} / \mathrm{E}^{\prime}$ ratio was also calculated as load-independent marker of ventricular diastolic relaxation [12]. Mitral regurgitation (MR) severity was graded according to the current guidelines [13]. LV responders to CRT were defined as those patients who presented a reduction of $>10 \%$ in LVESVi at 6-month follow-up, whereas those with a lesser degree of reduction of $10 \%$ were defined as nonresponders [14].

\subsubsection{LA evaluation}

Anteroposterior LA diameter (iLAd) was assessed by M-mode scan in parasternal long axis view. LA volumes and LA emptying fraction were calculated from the apical two- and four-chamber images using the biplane Simpson's rule and were indexed to body surface area [8].

In order to evaluate the complexity of LA function we specified three atrial phases. The first is the reservoir phase: during ventricular systole and isovolumic relaxation blood arrive into LA from pulmonary venous return; in this phase LA stores energy in the form of pressure. The second is the passive phase: after mitral valve opening, in the early phase of ventricular diastole, the LA transfers blood into the LV because of pressure gradient, so blood flows passively from the pulmonary veins into the left ventricle. Finally in the contractile phase the LA systole serves to augment the LV stroke volume.

The following left atrial volume (LAV) parameters were recorded in order to completely describe the LA contribution:

- Maximal LAV at ventricular end-systole where LA size is maximal (iLAVmax),

- LAV just before atrial systole (iLAVpre),

- Minimal LAV after atrial systole (iLAVpost).

Moreover, left atrial emptying fractions (LAEF) were derived from LA volumes and calculated as follows:

- Total LA emptying volume (LAEFtot): [(iLAVmax - iLAVpost) / iLAVmax] $\times 100$,

- LA passive emptying volume (LAEFpas): [(iLAVmax - iLAVpre) / iLAVmax $] \times 100$,

- LA active emptying volume (LAEFcon): [(iLAVpre - iLAVpost) / iLAVpre] $\times 100$.

\subsection{Statistical analysis}

Statistical analysis was conducted using Package for Social Sciences version 17.0 for Windows (SPSS Inc., Chicago, IL, USA) and Medcalc 7.3. Comparison of the continuous parametric variables between baseline and 6-month follow-up was performed using a paired sample $t$ test or the $\chi^{2}$ test for the ordinal variables. Unpaired $t$ test was used to compare the echocardiographic parameters between responders and nonresponders. Logistic regression analysis was used to identify univariate and multivariable predictors of CRT response. For the univariable models, $p$-value $\leq 0.10$ was considered significant and the corresponding variable was included in the multivariable model. The odds ratio (OR) and 95\% confidence interval $(\mathrm{CI})$ are shown. All parametric data were expressed as mean $\pm \mathrm{SD}$. A $p$-value $<0.05$ was considered statistically significant.

\section{Results}

\subsection{Repeatability and reproducibility of measurements}

Reproducibility of our laboratories has been published previously [15]. Briefly, for iLAVmax assessment intra-observer variability was $4.8 \pm 1.6 \%$ and inter-observer was $6.7 \pm 3.2 \%$; for LVEDVi, intra-observer variability was $5.7 \pm 4.4 \%$ and inter-observer was $8.3 \pm 4.6 \%$ both indicating outstanding reliability.

\subsection{Study population}

Baseline characteristics of the overall population are presented in Table 1. Ischemic heart disease was present in $44 \%$ of patients; $46 \%$ of patients were in NYHA class II, $50 \%$ in class III and $4 \%$ in class IV. Almost all patients were treated with a beta-blocker (92\%) and with ACEinhibitor or ARB (97\%), $79 \%$ of patients had a diuretics. All patients had severe LV dilation and eleven patients (21\%) presented severe MR.

\subsection{Clinical and echocardiographic improvement after CRT}

At 6-month follow-up, there was an improvement of clinical status for the overall population, namely NYHA functional class and Minnesota Living With Heart Failure QOL score. CRT resulted in a significant LA (mean iLAVmax from $55 \pm 16.4 \mathrm{ml} / \mathrm{m}^{2}$ to $50.3 \pm 19 \mathrm{ml} / \mathrm{m}^{2}, p 0.006$ ) and LV reverse remodeling (mean LVESVi from $94.7 \pm 52.1 \mathrm{ml} / \mathrm{m}^{2}$ to $72.9 \pm 54.1 \mathrm{ml} / \mathrm{m}^{2}, p<0.001$ ) leading to an increase in both systolic function. Moreover, a significant reduction of MR and QRS duration was observed (Table 2). Biventricular pacing percentage was accurately assessed through device counters' analysis performed at the 6-month visit: a percentage of biventricular pacing $>85 \%$ was observed in all patients and no atrial or ventricular arrhythmias were recorded. During follow-up no patient died or was hospitalized for worsening heart failure as well for device-related problems.

Table 1

Demographic and baseline criteria $(\mathrm{n}=52)$.

\begin{tabular}{lll}
\hline Age (yrs) & & $67.1 \pm 10.2$ \\
Gender (M/F) & & $38 / 14$ \\
Systemic hypertension & & $15(29 \%)$ \\
Diabetes mellitus & & $14(27 \%)$ \\
GFR (ml/min/1.73 m²) & $68 \pm 21$ \\
PR interval (ms) & & $202 \pm 30$ \\
Ischemic etiology & & $23(44 \%)$ \\
NYHA functional class: & II & \\
& III & $24(46 \%)$ \\
& IV & $26(50 \%)$ \\
Medications: & & $2(4 \%)$ \\
& Beta-blockers & $48(92 \%)$ \\
& ACE inhibitors/ARBs & $50(97 \%)$ \\
& Diuretics & $41(79 \%)$ \\
\hline
\end{tabular}


Table 2

Clinical status and echocardiographic parameters at baseline and 6-month follow-up.

\begin{tabular}{lccc}
\hline & $\begin{array}{l}\text { All patients }(\mathrm{n}=52) \\
\text { baseline }\end{array}$ & $\begin{array}{l}\text { All patients }(\mathrm{n}=52) \\
\text { 6-month follow-up }\end{array}$ & $p$ value \\
\hline QRS interval $(\mathrm{ms})$ & $166 \pm 20$ & $137 \pm 22$ & $<0.001$ \\
NYHA functional class & $2.6 \pm 0.6$ & $1.9 \pm 0.6$ & $<0.001$ \\
Qol score & $36 \pm 17$ & $21 \pm 16$ & $<0.001$ \\
LVEDVi $\left(\mathrm{ml} / \mathrm{m}^{2}\right)$ & $121.7 \pm 53.5$ & $103.6 \pm 61.8$ & $<0.001$ \\
LVESVi $\left(\mathrm{ml} / \mathrm{m}^{2}\right)$ & $94.7 \pm 52.1$ & $72.9 \pm 54.1$ & $<0.001$ \\
LVEF $(\%)$ & $22.2 \pm 9.3$ & $29.6 \pm 10.1$ & $<0.001$ \\
Mitral regurgitation: & & & \\
$\quad$ EROA $\left.(\mathrm{mm})^{2}\right)$ & $27 \pm 3$ & $15 \pm 3$ & $<0.001$ \\
R Vol $(\mathrm{ml})$ & $42 \pm 4$ & $22 \pm 3$ & $<0.001$ \\
E/A & $1.46 \pm 0.32$ & $1.02 \pm 0.24$ & 0.01 \\
E/E' & $21 \pm 5$ & $15 \pm 8$ & $<0.001$ \\
iLA diameter $\left(\mathrm{mm} / \mathrm{m}^{2}\right)$ & $34.8 \pm 4.1$ & $34.2 \pm 3.9$ & 0.34 \\
iLAVmax $\left(\mathrm{ml} / \mathrm{m}^{2}\right)$ & $55.8 \pm 16.4$ & $50.3 \pm 19$ & 0.006 \\
iLAVpre $\left(\mathrm{ml} / \mathrm{m}^{2}\right)$ & $47 \pm 15.2$ & $41.4 \pm 17.4$ & 0.003 \\
iLAVpost $\left(\mathrm{ml} / \mathrm{m}^{2}\right)$ & $38.7 \pm 15.3$ & $30.3 \pm 18$ & $<0.001$ \\
LAEFtot $(\%)$ & $31.9 \pm 13.7$ & $42.8 \pm 16$ & $<0.001$ \\
LAEFpas $(\%)$ & $15.8 \pm 9.2$ & $18.3 \pm 10.4$ & 0.17 \\
LAEFcon $(\%)$ & $19.1 \pm 13.1$ & $29.5 \pm 18.4$ & 0.001 \\
\hline
\end{tabular}

$\mathrm{EROA}=$ effective regurgitant orifice area; $\mathrm{R}$ Vol = regurgitant volume.

\subsection{Responders and nonresponders to CRT}

At 6-month follow-up, thirty three (63\%) patients showed a reduction of $>10 \%$ in LVESVi (mean reduction: $-28.5 \pm 33.7 \mathrm{ml} / \mathrm{m}^{2}$ ) and were therefore classified as responders to CRT; nineteen (37\%) patients had a reduction of $\leq 10 \%$ in LVESVi and were therefore classified as nonresponders to CRT. At baseline, clinical characteristics as well as LV volumes were similar between responders and nonresponders (Table 3). However, baseline LAV measurements were significantly associated with the extent of LV reverse remodeling (Table 3): CRT responders displayed a significantly lower baseline iLAVmax, iLAVpre and iLAVpost compared to CRT nonresponders. Baseline LAEFtot and LAEFcon in the responders were greater, although not statistically significant. However, baseline LAEFpas and baseline LA diameter were similar between responders and nonresponders.

Finally, the prevalence of baseline echocardiography dyssynchrony was higher in the responders, although this difference was not statistically significant.

Table 3

Baseline characteristics of responders vs nonresponders to CRT

\begin{tabular}{lccc}
\hline & $\begin{array}{c}\text { Responders } \\
(\mathrm{n}=33)\end{array}$ & $\begin{array}{l}\text { Nonresponders } \\
(\mathrm{n}=19)\end{array}$ & $p$ value \\
\hline Age (yrs) & $67.6 \pm 11.4$ & $66.5 \pm 10.2$ & 0.54 \\
QRS interval (ms) & $169 \pm 19$ & $162 \pm 21$ & 0.26 \\
NYHA functional class & $2.63 \pm 0.6$ & $2.59 \pm 0.6$ & 0.81 \\
Qol score & $36 \pm 19$ & $37 \pm 18$ & 0.84 \\
LVEDVi (ml/m $\left.{ }^{2}\right)$ & $115.3 \pm 47.2$ & $135.8 \pm 47.3$ & 0.19 \\
LVESVi $\left(\mathrm{ml} / \mathrm{m}^{2}\right)$ & $90 \pm 42$ & $103.9 \pm 64.6$ & 0.43 \\
LVEF $(\%)$ & $22.3 \pm 9.3$ & $25.8 \pm 9.3$ & 0.22 \\
IVMD $(\mathrm{ms})$ & $52 \pm 34$ & $43 \pm 31$ & 0.08 \\
Ts-LS (ms) & $49 \pm 37$ & $42 \pm 42$ & 0.12 \\
Mitral regurgitation & & & \\
$\quad$ EROA (mm & & \\
R Vol $(\mathrm{ml})$ & $26 \pm 2$ & $29 \pm 3$ & 0.45 \\
E/A & $1.44 \pm 0.29$ & $1.46 \pm 0.24$ & 0.65 \\
E/E' & $20 \pm 6$ & $21 \pm 8$ & 0.48 \\
iLA diameter $\left(\mathrm{mm} / \mathrm{m}^{2}\right)$ & $34.3 \pm 4.3$ & $35.3 \pm 4.3$ & 0.72 \\
iLAVmax $\left(\mathrm{ml} / \mathrm{m}^{2}\right)$ & $50.2 \pm 14.1$ & $65.8 \pm 15.7$ & 0.43 \\
iLAVpre $\left(\mathrm{ml} / \mathrm{m}^{2}\right)$ & $42.5 \pm 13.4$ & $55.1 \pm 15.1$ & 0.001 \\
iLAVpost $\left(\mathrm{ml} / \mathrm{m}^{2}\right)$ & $34.1 \pm 13.8$ & $46.8 \pm 14.9$ & 0.005 \\
LAEFtot $(\%)$ & $33.9 \pm 12.6$ & $28.4 \pm 15.3$ & 0.005 \\
LAEFpas $(\%)$ & $15.7 \pm 8.2$ & $16.1 \pm 11.1$ & 0.19 \\
LAEFcon $(\%)$ & $21.6 \pm 12.7$ & $14.8 \pm 12.9$ & 0.09 \\
\hline
\end{tabular}

$\overline{\mathrm{IVMD}}=$ Inter-ventricular mechanical delay; Ts-LS = time delay between time-to-peak systolic velocities at the LV basal lateral and septal segments.

$\mathrm{EROA}=$ effective regurgitant orifice area; $\mathrm{R} \mathrm{Vol}=$ regurgitant volume.

\subsection{Left atrial volumes as predictors of CRT response}

Differences between baseline LA volumes suggested their predictive role for CRT response. Moreover, a linear correlation was found between baseline LA volumes and LV reverse remodeling. In particular, iLAVmax showed the best relationship for LVESVi reduction (Fig. 1). A similar correlation resulted for LAEFtot and LAEFcon; conversely, no correlation was found between baseline iLAd and LAEFpas for LVESVi reduction. Univariate regression analysis confirmed these results: IVMD, Ts-LS, QRS interval and smaller LA volumes were found to be predictors of CRT response (Table 4). Variables in the univariate analysis with $p \leq 0.10$ were included in the multivariable model. iLAVmax resulted the only parameter significantly and independently predictive for CRT response $(p=0.007)$.

To further investigate the ability of iLAVmax to predict LV reverse remodeling, receiving operating characteristic (ROC) curve analysis was performed (Fig. 2). The areas under the curve (AUC) for the ROC curve with iLAVmax used to predict LV reverse remodeling was 0.767 (95\% CI, $0.618-0.916 ; p<0.01)$. Optimal sensitivity and specificity were obtained at a cutoff level of $58 \mathrm{ml} / \mathrm{m}^{2}$ (71\% and $74 \%$ respectively). An iLAVmax value of $50 \mathrm{ml} / \mathrm{m}^{2}$ was $88 \%$ sensitive as predictor of CRT response, although specificity decreased to $57 \%$.

\section{Discussion}

In patients with HF and prolonged QRS duration, CRT provides clinical benefits and improvement of LV systolic function as a result of LV reverse remodeling [16-18]. Thus, there is a strong clinical mandate for the use of CRT in eligible patients that is supported by international practice guidelines [19]. Although most treated patients show a benefit from CRT, nonresponders of this therapy have been consistently observed in about one-third of patients [1].

Recently, several echocardiographic measurements of mechanical dyssynchrony have been proposed to identify responders to CRT before device implantation. However, the Predictors of Response to CRT (PROSPECT) trial reported that no single echocardiographic measurement of dyssynchrony can be recommended to improve patient selection for CRT [20]. Currently, we are looking for some pre-implantation clinical and echocardiographic parameters able to predict CRT response in order to optimize the decision making.

LA is an asymmetric thin-walled structure and its size may increase with pathological LV filling pressure, providing a simple noninvasive assessment of the degree of LV diastolic dysfunction [21-24]. Recent data suggest LA dilatation as a predictor of common cardiovascular outcomes such as atrial fibrillation, congestive HF, cardiovascular death and stroke [4-6]. Nevertheless, the impact of LA size in patients with CRT has not been fully evaluated. Shen et al. retrospectively showed the pre-implantation LA enlargement as a predictor of mortality, but failed to assess the LA predictive role for LV reverse remodeling after CRT implantation [7]. Accordingly, our study is the first to prospectively demonstrate the predictive role of LA volume for CRT response and we should conclude that more advanced is HF and larger is LA size, therefore response to CRT will be unlikely. Furthermore, we propose an iLAVmax cutoff value of $58 \mathrm{ml} / \mathrm{m}^{2}$ beyond that low probability of CRT response is expected. This iLAVmax cutoff value is similar to $59 \mathrm{ml} / \mathrm{m}^{2}$ LAV index cutoff previously proposed by Shen et al.

Our study is the first to analyze the predictive role of several LA measurements for CRT response. LA dimension is usually measured at the ventricular end-systole from the parasternal long-axis view. However, it has been shown that LA antero-posterior diameter is an inaccurate representation of its size, because LA may not dilate equally along its three axes [25]. Recent data suggest that two-dimensionalderived LA volume provides a more accurate measure of LA true size. End-systolic LA volume from apical four-chamber view is the one routinely measured in clinical practice. Nevertheless, a comprehensive assessment of LA function as previously described is mandatory 


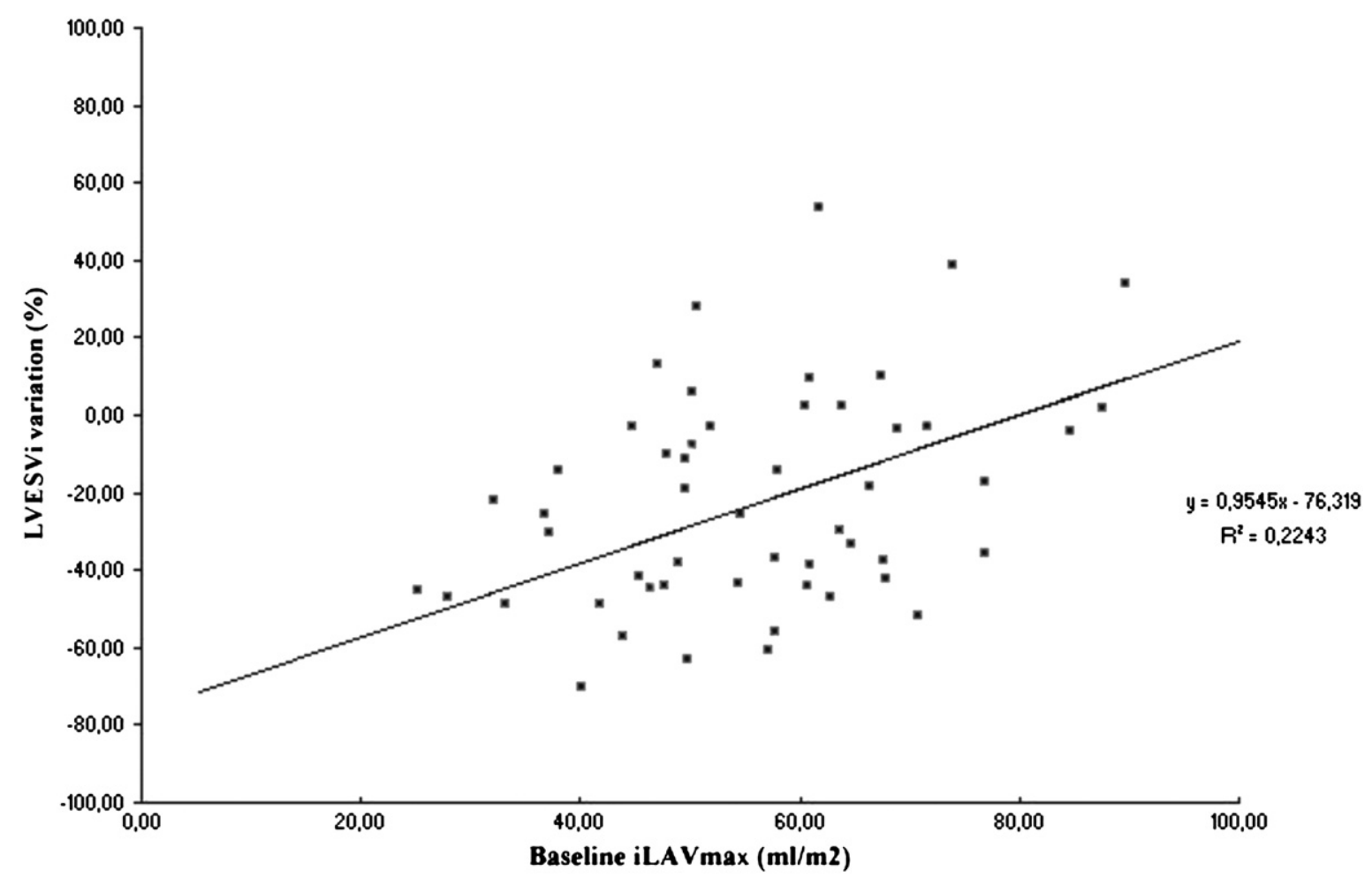

Fig. 1. Linear correlation between baseline iLAVmax and LV reverse remodeling.

[26-31]. Accordingly, we specified three LA phases: iLAVmax and LAEFtot represent the reservoir phase, iLAVpre and LAEFpas represent the conduit phase, iLAVpost and LAEFcon represent the contractile phase. We demonstrated the predictive role to CRT response for all LA volumes; the best linear correlation was found for iLAVmax, likely as expression of LV filling pressure over time. Indeed the contribution of LA phasic function to LV filling is dependent on LV diastolic properties: in the early diastolic dysfunction when abnormal relaxation occurs, the relative contribution of LA contractile function to LV filling increases, whereas the conduit function decreases. As LV filling pressure progressively increases, the LA volume increase to the limits of atrial preload

Table 4

Predictors of CRT response.

\begin{tabular}{llll}
\hline & OR & $95 \% \mathrm{CI}$ & $p$ value \\
\hline Age (1-year increase) & 1.02 & $0.96-1.08$ & 0.54 \\
Sex (male) & 0.85 & $0.21-3.37$ & 0.81 \\
Ischemic etiology & 0.75 & $0.22-2.49$ & 0.64 \\
QRS interval (ms) & 1.12 & $1.03-1.20$ & 0.09 \\
NYHA functional class & 1.13 & $0.42-3.06$ & 0.8 \\
Qol score & 1.52 & $0.96-2.4$ & 0.34 \\
LVEDVi (ml $\left./ \mathrm{m}^{2}\right)$ & 0.99 & $0.98-1.00$ & 0.24 \\
LVESVi (ml $\left./ \mathrm{m}^{2}\right)$ & 0.99 & $0.98-1.01$ & 0.37 \\
LVEF $(\%)$ & 0.94 & $0.90-0.98$ & 0.46 \\
IVMD (ms) & 1.11 & $1.01-1.22$ & 0.08 \\
Ts-LS (ms) & 1.06 & $1.02-1.10$ & 0.07 \\
Mitral regurgitation: EROA $\left(\mathrm{mm}^{2}\right)$ & 0.83 & $0.52-1.33$ & 0.44 \\
iLAd (mm/m ${ }^{2}$ ) & 1.17 & $0.25-5.54$ & 0.84 \\
iLAVmax (mm/ $\mathrm{m}^{2}$ ) & 0.93 & $0.89-0.98$ & 0.004 \\
iLAVpre (ml $\left./ \mathrm{m}^{2}\right)$ & 0.94 & $0.90-0.99$ & 0.011 \\
iLAVpost (ml $\left./ \mathrm{m}^{2}\right)$ & 0.94 & $0.89-0.99$ & 0.011 \\
LAEFtot $(\%)$ & 1.03 & $0.98-1.08$ & 0.18 \\
LAEFpas (\%) & 0.99 & $0.93-1.06$ & 0.89 \\
LAEFcon (\%) & 1.05 & $0.99-1.12$ & 0.11
\end{tabular}

IVMD = Inter-ventricular mechanical delay; Ts-LS = time delay between time-to-peak systolic velocities at the LV basal lateral and septal segments.

EROA $=$ effective regurgitant orifice area. reserve by the Frank-Starling mechanism. Conversely, LAEF is more representative of the intrinsic atrial function and so less connected to LV filling pressure, resulting worst related to CRT response.

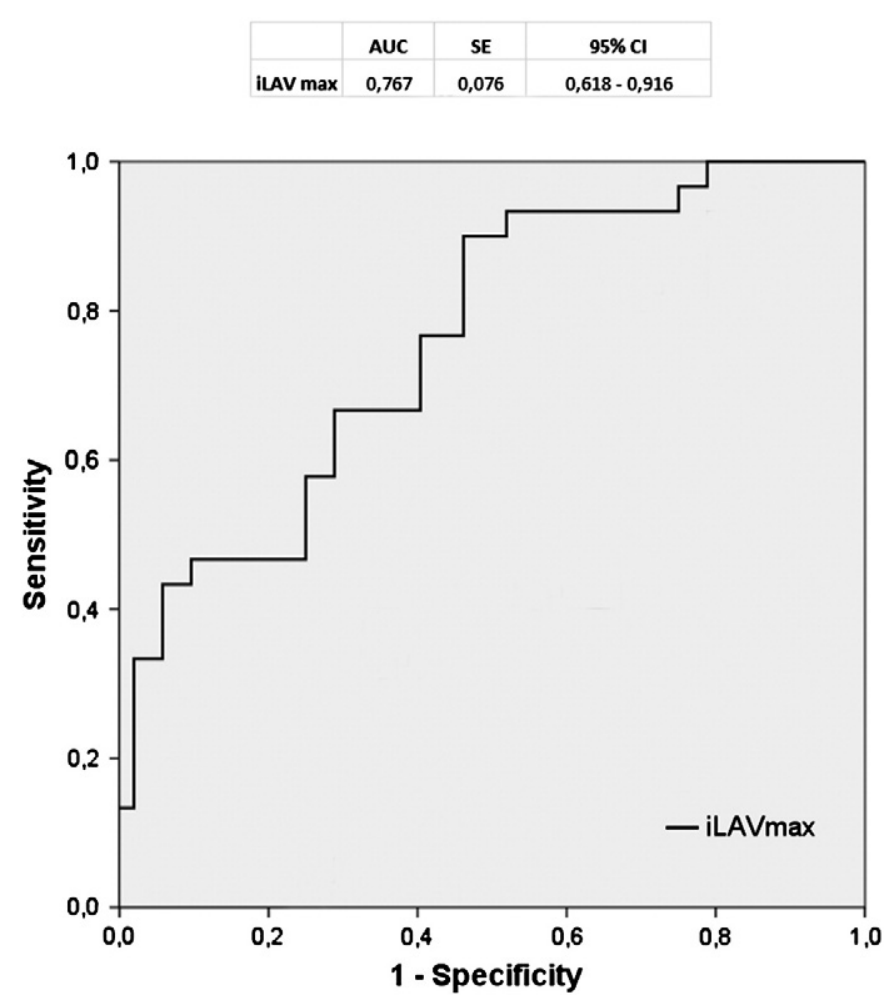

Fig. 2. Receiver operating characteristic curve of iLAVmax values as predictors of LV reverse remodeling. $\mathrm{AUC}=$ area under the curve; $\mathrm{SE}=$ standard error; $\mathrm{CI}=$ confidence interval. 


\subsection{Study limitation}

This is a prospective noninvasive study with a small sample size, investigating LA volume as a predictor of CRT response. Further large-scale, multicenter studies collecting follow-up data are required to confirm our results. Furthermore, we performed the LA evaluation just by volumes and empting fraction; however, we cannot deny that newer echocardiographic technologies such as three-dimensional speckle tracking might assess the LA function in a more proper way. Another potential source of bias is the lack of a central echo core-lab; nevertheless, this limitation is exceeded by the low variability between the investigators who performed echocardiograms.

\section{Conclusion}

Patients with small LA volume result as better responders to CRT. In particular, iLAVmax is an independent predictor of LV reverse remodeling and allows to identify best candidates for CRT.

\section{Acknowledgments}

The authors of this manuscript have certified that they comply with the Principles of Ethical Publishing in the International Journal of Cardiology.

\section{References}

[1] Cleland JG, Daubert JC, Erdmann E, et al. Cardiac Resynchronization-Heart Failure (CARE-HF) Study Investigators. The effect of cardiac resynchronization on morbidity and mortality in heart failure. N Engl J Med 2005;352:1539-49.

[2] Moss AJ, Hall WJ, Cannom DS, et al. Cardiac-resynchronization therapy for the prevention of heart-failure events. N Engl J Med 2009;361:1329-38.

[3] Abraham WT, Fisher WG, Smith AL, et al. Cardiac resynchronization in chronic heart failure. N Engl J Med 2002;346:1845-53.

[4] Sabharwal N, Cemin R, Rajan K, Hickman M, Lahiri A, Senior R. Usefulness of left atrial volume as a predictor of mortality in patients with ischemic cardiomyopathy. Am J Cardiol 2004;94:760-3.

[5] Benjamin EJ, D'Agostino RB, Belanger AJ, Wolf PA, Levy D. Left atrial size and the risk of stroke and death. The Framingham Heart-Study. Circulation 1995;92:835-41.

[6] Lim TK, Dwivedi G, Hayat S, Majumdar S, Senior R. Independent value of left atrial volume index for the prediction of mortality in patients with suspected heart failure referred from the community. Heart 2009;95:1172-8.

[7] Shen X, Nair CK, Holmberg MJ, et al. Impact of left atrial volume in prediction of outcome after cardiac resynchronization therapy. Int J Cardiol 2011;152:13-7.

[8] Du Bois D, Du Bois EF. A formula to estimate the approximate surface area if height and weight be known. Nutrition 1989;5:303-11.

[9] Bax JJ, Marwick TH, Molhoek SG, et al. Left ventricular dyssynchrony predicts benefit of cardiac resynchronization therapy in patients with end-stage heart failure before pacemaker implantation. Am J Cardiol 2003:92:1238-40.

[10] Quinones MA, Otto CM, Stoddard M, Waggoner A, Zoghbi WA. Recommendations for quantification of Doppler echocardiography: a report from the Doppler quantification Task force of the Nomenclature and Standards Committe of the American Society of Echocardiography. J Am Soc Echocardiogr 2002;15:167-84

[11] Yu CM, Sanderson JE, Marwick OhJK. Tissue Doppler imaging a new prognosticator for cardiovascular disease. J Am Coll Cardiol 2007;49:1903-14

[12] Ommen SR, Nishimura RA, Appleton CP, et al. Clinical utility of Doppler echocardiography and tissue Doppler imaging in the estimation of left ventricular filling pressures: a comparative simultaneous Doppler-catheterization study. Circulation 2000;102:1788-94

[13] Lancellotti P, Moura L, Pierard LA, et al. European Association of Echocardiography recommendations for the assessment of valvular regurgitation. Part 2: mitral and tricuspid regurgitation (native valve disease). Eur J Echocardiogr 2010:11:307-32

[14] Davis DR, Krahn AD, Tang AS, et al. Long-term outcome of cardiac resynchronization therapy in patients with severe congestive heart failure. Can J Cardiol 2005;21: 413-7.

[15] Malagoli A, Rossi L, Franchi F, et al. Effect of cardiac resynchronization therapy on left atrial reverse remodeling: Role of echocardiographic AV delay optimization. Int J Cardiol Aug 20 2013;167(4):1456-60.

[16] Cazeau S, Leclercq C, Lavergne T, et al. Multisite Stimulation in Cardiomyopathies (MUSTIC) Study Investigators. Effects of multisite biventricular pacing in patients with heart failure and intraventricular conduction delay. N Engl J Med 2001;344: 873-80.

[17] Auricchio A, Stellbrink C, Block M, et al. Effect of pacing chamber and atrioventricular delay on acute systolic function of paced patients with congestive heart failure. Circulation 1999;99:2993-3001.

[18] Auricchio A, Stellbrink C, Sack S, et al. Long-term clinical effect of hemodynamically optimized cardiac resynchronization therapy in patients with heart failure and ventricular conduction delay. J Am Coll Cardiol 2002;39:2026-33.

[19] Dickstein K, Vardas PE, Auricchio A, et al. 2010 focused update of European Society of Cardiology guidelines on device therapy in heart failure. Eur Heart J 2010;31: 2677-87.

[20] Chung ES, Leon AR, Tavazzi L, et al. Results of the Predictors of Response to CRT (PROSPECT) Trial. Circulation 2008;117:2608-16.

[21] Hurrell DG, Nishimura RA, Ilstrup DM, Appleton CP. Utility of preload alteration in assessment of left ventricular filling pressure by Doppler echocardiography: a simultaneous catheterization and Doppler echocardiographic study. J Am Coll Cardiol 1997;30:459-67.

[22] Appleton CP, Galloway JM, Gonzalez MS, Gaballa M, Basnight MA. Estimation of left ventricular filling pressures using two-dimensional and Doppler echocardiography in adult patients with cardiac disease. Additional value of analyzing left atrial size, left atrial ejection fraction and the difference in duration of pulmonary venous and mitral flow velocity at atrial contraction. J Am Coll Cardiol 1993;22: 1972-82.

[23] Simek CL, Feldman MD, Haber HL, Wu CC, Jayaweera AR, Kaul S. Relationship between left ventricular wall thickness and left atrial size: comparison with other measures of diastolic function. J Am Soc Echocardiogr 1995;8:37-47.

[24] Tsang TS, Barnes ME, Gersh BJ, Bailey KR, Seward JB. Left atrial volume as a morphophysiologic expression of left ventricular diastolic dysfunction and relation to cardiovascular risk burden. Am J Cardiol 2002;90:1284-9.

[25] Lester SJ, Ryan EW, Schiller NB, Foster E. Best method in clinical practice and in research studies to determine left atrial size. Am J Cardiol 1999;84:829-32.

[26] Braunwald E, Frahm C, Jr Ross. Studies on Starling's law of the heart, IV: observations on the hemodynamic functions of the left atrium in man. Circulation 1961;24: 633-42.

[27] Spencer KT, Mor-Avi V, Gorcsan IJ, et al. Effects of aging on left atrial reservoir, conduit, and booster pump function: a multiinstitution acoustic quantification study. Heart 2001;85:272-7.

[28] Thomas L, Levett K, Boyd A, Leung DYC, Schiller NB, Ross DL. Changes in regional left atrial function with aging: evaluation by Doppler tissue imaging. Eur J Echocardiogr 2003;4:92-100.

[29] Nikitin NP, Witte KKA, Thackray SDR, Goodge LJ, Clark AL, Cleland JGF. Effect of age and sex on left atrial morphology and function. Eur J Echocardiogr 2003;4: 36-42.

[30] Stefanadis C, Dernellis J, Toutouzas P. A clinical appraisal of left atrial function. Eur Heart J 2001;22:22-36.

[31] Lang RM, Bierig M, Devereux RB, et al. European Association of Echocardiography. Recommendations for chamber quantification: a report from the American Society of Echocardiography's Guidelines and Standards Committee and the Chamber Quantification Writing Group, Developed in Conjunction with the European Association of Echocardiography, a Branch of the European Society of Cardiology. J Am Soc Echocardiogr 2005;18:1440-63. 